\title{
Moi, jouer ? Observations sur l'acquisition du français en tant que langue seconde chez des élèves anglodominants au sein d'une classe de maternelle francophone Me, play? Observations on the acquisition of French as a second language by Anglo-dominant students in a Francophone kindergarten class \\ ¿Yo, jugar? Observaciones sobre la adquisición del francés en tanto que segunda lengua entre los alumnos anglo-dominantes en una clase de preescolar francófona
}

\section{Rachelle Gauthier et Marianne Cormier}

Volume 45, numéro 2, automne 2017

La littératie, tout au long de la vie

URI : https://id.erudit.org/iderudit/1043528ar

DOI : https://doi.org/10.7202/1043528ar

Aller au sommaire du numéro

\section{Éditeur(s)}

Association canadienne d'éducation de langue française

ISSN

1916-8659 (numérique)

Découvrir la revue

Citer cet article

Gauthier, R. \& Cormier, M. (2017). Moi, jouer ? Observations sur l'acquisition du français en tant que langue seconde chez des élèves anglodominants au sein d'une classe de maternelle francophone. Éducation et francophonie, 45(2),

47-66. https://doi.org/10.7202/1043528ar

\section{Résumé de l'article}

Actuellement, l'école francophone en milieu minoritaire est aux prises avec de nombreux enjeux sociodémographiques alarmants qui font en sorte que le nombre d'élèves admissibles à l'école de la minorité diminue progressivement et qu'un enfant admissible sur deux n'a pas le français comme langue maternelle (Landry, 2010). De ce fait, les conseils scolaires francophones à travers le pays se heurtent au défi de maximiser la participation à l'école de langue française, ce qui se traduit par une diversité linguistique grandissante (Cormier, 2015; Farmer, 2008). Il apparaît donc essentiel de mieux comprendre les interactions entre le français et l'anglais dans les pratiques langagières des élèves. Nous rapportons ici les détails d'une étude qualitative, menée entre novembre 2015 et avril 2016 dans trois classes de maternelle francophones, qui cherchait à mieux comprendre les comportements langagiers des élèves dans des contextes éducatifs francophones où la francisation était présente. L'analyse des données provenant d'une école qui se situe dans un contexte de vitalité forte indique que les élèves anglodominants cheminaient bien à travers les stades d'acquisition d'une deuxième langue décrits par Tabors (2008) et qu'ils intégraient progressivement le français dans leurs locutions. Pour leur part, les élèves francodominants étaient portés, en novembre, à choisir l'anglais dans leurs échanges avec les élèves anglodominants, tandis qu'ils misaient fortement sur le français en avril.
Tous droits réservés ( Association canadienne d'éducation de langue française, 2017
Ce document est protégé par la loi sur le droit d'auteur. L'utilisation des services d’Érudit (y compris la reproduction) est assujettie à sa politique d'utilisation que vous pouvez consulter en ligne. 


\section{Moi, jouer? Observations sur l'acquisition du français en tant que langue seconde chez des élèves anglodominants au sein d'une classe de maternelle francophone}

\section{Rachelle GAUTHIER}

Université de Moncton, Nouveau-Brunswick, Canada

\section{Marianne CORMIER}

Université de Moncton, Nouveau-Brunswick, Canada

\section{RÉSUMÉ}

Actuellement, l'école francophone en milieu minoritaire est aux prises avec de nombreux enjeux sociodémographiques alarmants qui font en sorte que le nombre d'élèves admissibles à l'école de la minorité diminue progressivement et qu'un enfant admissible sur deux n'a pas le français comme langue maternelle (Landry, 2010). De ce fait, les conseils scolaires francophones à travers le pays se heurtent au défi de maximiser la participation à l'école de langue française, ce qui se traduit par une diversité linguistique grandissante (Cormier, 2015; Farmer, 2008). Il apparaît donc essentiel de mieux comprendre les interactions entre le français et l'anglais 
dans les pratiques langagières des élèves. Nous rapportons ici les détails d'une étude qualitative, menée entre novembre 2015 et avril 2016 dans trois classes de maternelle francophones, qui cherchait à mieux comprendre les comportements langagiers des élèves dans des contextes éducatifs francophones où la francisation était présente. L'analyse des données provenant d'une école qui se situe dans un contexte de vitalité forte indique que les élèves anglodominants cheminaient bien à travers les stades d'acquisition d'une deuxième langue décrits par Tabors (2008) et qu'ils intégraient progressivement le français dans leurs locutions. Pour leur part, les élèves francodominants étaient portés, en novembre, à choisir l'anglais dans leurs échanges avec les élèves anglodominants, tandis qu'ils misaient fortement sur le français en avril.

\section{ABSTRACT}

\section{Me, play? Observations on the acquisition of French as a second language by Anglo-dominant students in a Francophone kindergarten class}

Rachelle GAUTHIER, University of Moncton, New Brunswick, Canada

Marianne CORMIER, University of Moncton, New Brunswick, Canada

Currently, Francophone minority schools face many alarming sociodemographic issues that make the number of students eligible for minority schools progressively lower, and one in two eligible children do not have French as mother tongue (Landry, 2010). As a result, Francophone school boards across the country are faced with the challenge of maximizing participation in French-language schools, resulting in increasing linguistic diversity (Cormier 2015, Farmer 2008). It therefore seems essential to better understand the interactions between French and English in the language practices of students. Here we report the details of a qualitative study conducted between November 2015 and April 2016 in three Francophone kindergarten classes, which sought to better understand the language behaviors of students in Francophone educational contexts where Francization was present. Analysis of data from a school in a context of strong vitality indicates that Anglo-dominant students were doing well through the stages of second language acquisition described by Tabors (2008) and that they were gradually integrating French into their speech. For their part, Franco-dominant students were asked in November to choose English in their exchanges with Anglo-dominant students, while they counted strongly on French in April. 


\section{RESUMEN}

\section{¿Yo, jugar? Observaciones sobre la adquisición del francés en tanto que segunda lengua entre los alumnos anglo-dominantes en una clase de preescolar francófona}

Rachelle GAUTHIER, Universidad de Moncton, Nuevo-Brunswick, Canadá

Marianne CORMIER, Universidad de Moncton, Nuevo-Brunswick, Canadá

Hoy en día, la escuela francófona en medio minoritario se enfrenta con numerosos desafíos sociodemográficos alarmantes que prevén que el número de alumnos admisibles a la escuela minoritaria disminuye progresivamente y que uno de cada dos niños admisibles no tiene el francés como lengua materna (Landry, 2020). En consecuencia, los consejos escolares francófonos a través del país se enfrentan al reto de maximizar la participación a la escuela de lengua francesa, lo que se traduce en una diversidad lingüística creciente (Cormier, 2015; Farmer, 2008). Parece pues esencial de conocer más a fondo las interacciones entre el francés y el inglés en las prácticas lingüísticas de los alumnos. En el presente artículo presentamos los detalles de un estudio cualitativo, realizado entre noviembre 2015 y abril 2016 en tres clases de preescolar francófonas, que trataba de conocer más cabalmente los comportamientos lingüísticos de los alumnos en contextos educativos francófonos en donde se daba el aprendizaje del francés. El análisis de los datos provenientes de una escuela que se encuentra en un contexto de vitalidad fuerte indica que los alumnos anglodominantes pasaban bien los estadios de adquisición de una segunda lengua, descritos por Tabors (2008) y que integraban progresivamente el francés en sus locuciones. Por su parte, los alumnos franco-dominantes tenían la tendencia, en noviembre, a escoger el inglés en sus intercambios con los alumnos anglo-dominantes, pero en abril, se aventuraban fuertemente a utilizar el francés.

\section{MISE EN CONTEXTE}

En raison du caractère double de sa mission, qui vise une éducation de haute qualité en français et l'épanouissement de la communauté (Arsenault-Cameron, 2000), l'école francophone en milieu minoritaire est devenue «l'institution la plus importante pour la survie de la minorité linguistique officielle» (Arsenault-Cameron, 2000). Lieu de socialisation et d'autonomisation pour les élèves, l'école francophone est depuis longtemps considérée comme la pierre angulaire du développement de la communauté minoritaire (Commissaire aux langues officielles, 2000; Cormier, 2015; Landry, 2003). Toutefois, cette institution est aux prises avec des enjeux sociodémographiques qui rendent difficile l'atteinte de sa mission. L'urbanisation, une proportion 
décroissante de la population francophone vis-à-vis de la majorité, le vieillissement de la population et un taux croissant d'exogamie ${ }^{1}$, tous ces éléments sont des «tendances lourdes» (Landry, Allard et Deveau, 2010) qui contribuent à la fragilité des communautés. Ces tendances font en sorte que le nombre d'élèves admissibles à l'école de la minorité diminue progressivement et qu'un enfant admissible sur deux n'a pas le français comme langue maternelle. Par ailleurs, la moitié des enfants admissibles à l'école de la minorité fréquentent celle-ci (Landry, 2010).

Devant ces enjeux considérables, les conseils scolaires francophones du pays doivent relever le défi de maximiser la participation à l'école de langue française tout en protégeant l'espace francophone. Non seulement ces défis se posent à tous les paliers du système scolaire, mais ils occasionnent une confrontation d'idées et d'opinions diverses chez les acteurs scolaires. Dans une perspective systémique, on se doit d'agir en attirant des effectifs scolaires, car il est plausible qu'après de longues luttes pour des écoles de langue française les communautés voient leurs écoles de plus en plus vides, ce qui rendrait impossible la réalisation de leur mission (Landry, 2010). De ce fait, des pratiques de marketing visant les couples exogames (Martel, 2001; Landry, 2010), de même qu'un assouplissement des critères d'admission aux écoles, jettent la base d'une vision hétérogène et pluraliste de l'école francophone et se traduisent par une diversification de l'école sur les plans linguistique et culturel (Farmer, 2008; Gallant et Denis, 2008; Gérin-Lajoie, 2006; Heller, 2011). Entre autres, cette situation crée des contextes scolaires où les élèves francophones (locuteurs L1) et les élèves anglodominants (apprenants L2) se côtoient dans les salles de classe.

Dans une perspective locale, en ce qui concerne les écoles, on perçoit que cette diversification complexifie la vie quotidienne (Gilbert, Letouzé, Thériault et Landry, 2004). Cormier (2015) a noté des sentiments d'ambivalence chez certains membres du personnel enseignant face à la présence d'élèves anglodominants dans leurs classes. Ces personnes s'expriment sur la fréquentation de l'école par ces élèves et donnent une voix à l'idée répandue selon laquelle ils pourraient " angliciser l'école et [...] menacer l'espace homogène francophone que l'on souhaite mettre en place pour contrebalancer l'anglodominance du milieu» (Cormier et Lowe, 2010, p. 225). Ce constat, souvent répété dans les médias traditionnels et à travers les réseaux de médias sociaux (voir, par exemple, Chandler, 2017), révèle une vision plutôt protectionniste du système scolaire francophone et rappelle une époque où l'école de langue française présentait une composition scolaire homogène (Gérin-Lajoie, 2006; Heller, 2011).

À la lumière de cet affrontement d'idées, qu'en est-il des élèves qui sont au centre de ce tiraillement idéologique? L'exogamie a été examinée sur le plan sociétal (Landry, 2003, 2006; Landry et Allard, 1997) ainsi qu'à travers les perceptions d'acteurs scolaires

1. Dans la plupart des cas en milieu minoritaire au Canada, un couple exogame est formé d'un parent francophone et d'un parent anglophone (Landry, 2003; Rocque, 2010-2011). 
et de parents (Rocque, 2006a, 2006b, 2010-2011). Cependant, rares sont les études qui privilégient les comportements et les perspectives des enfants anglodominants nés de parents ayants droit dans le but d'éclairer les processus d'acquisition du français en tant que langue seconde dans le contexte des écoles du milieu minoritaire francophone.

Dans l'ensemble, lorsqu'on discute de l'acquisition du français au sein des écoles de langue française, on fait référence à la francisation, renvoyant ainsi à un grand éventail d'approches et de stratégies qui visent l'acquisition des habiletés langagières nécessaires pour entreprendre des études dans une école française. Bien que la francisation existe dans les écoles de façon formelle et informelle depuis les années 1980 (Conseil des ministres de l'Éducation [Canada], 2002), elle doit encore faire l'objet d'un nombre significatif de recherches empiriques. En outre, les recherches menées à ce sujet jusqu'à maintenant ont mis l'accent sur la prestation des programmes de francisation ainsi que sur les perceptions d'intervenants scolaires et de parents (Cormier et Lowe, 2010; Cormier, Bourque et Jolicoeur, 2014; Cormier, 2015). En raison de ce déficit en recherche, nous en savons très peu sur le processus de francisation tel qu'il est vécu par les élèves (Cormier, Bourque et Jolicoeur, 2014).

Dans le but de contribuer à la production de nouvelles connaissances au sujet de la francisation, nous présentons ici les détails d'une étude qualitative qui mise sur les échanges interpersonnels entre des élèves anglodominants et leurs camarades de classe francodominants. Plus précisément, nous cherchons à mieux comprendre les comportements langagiers des élèves qui sont dans des classes de langue française où la francisation est présente.

Puisque, de façon générale, la francisation correspond à l'apprentissage du français dans le contexte des écoles françaises en milieu minoritaire (Conseil des ministres de l'Éducation [Canada], 2000), nous nous sommes tournées vers les experts de l'acquisition d'une langue seconde (L2) afin d'établir le cadre théorique de ce projet. En choisissant des théories appartenant au domaine du bilinguisme, notre intention était de nous informer sur le processus d'acquisition d'une L2 (second language acquisition) et de voir comment ces théories pouvaient s'appliquer au contexte des écoles de langue française. Toutefois, il apparaît nécessaire d'apporter un bémol en ce qui a trait aux comparaisons entre la francisation et le processus de l'acquisition d'une L2.

D'abord, la majorité des études liées à l'acquisition d'une langue seconde s'effectuent dans un contexte majoritaire, où les apprenants sont des locuteurs d'une langue souvent très minorisée et peu parlée dans la communauté, qui apprennent la langue de la majorité à l'école. Dans de tels contextes, les enfants comprennent rapidement l'importance de l'apprentissage de cette langue pour l'insertion sociale dans l'école et la communauté. À l'opposé, pour les élèves anglodominants qui fréquentent une école de langue française, le français peut sembler moins pertinent, puisque 
leur langue maternelle, l'anglais, est d'usage partout dans le contexte social. Dans ce cas, c'est donc l'anglais, et non le français, qui est valorisé et renforcé par l'environnement de l'enfant. Cette situation rend difficile la création d'un environnement francophone authentique et riche, mais c'est exactement ce que l'école doit créer afin de réaliser sa double mission. En gardant en tête cette distinction importante, nous avons procédé en envisageant la création de liens théoriques possibles entre les deux situations d'apprentissage.

\section{CADRE THÉORIQUE}

L'acquisition d'une langue seconde est un domaine de recherche axé sur les individus et les groupes qui acquièrent une langue seconde après avoir appris la première (Saville-Troike, 2006). À l'intérieur de ce domaine de recherche bien développé, plusieurs chercheurs de renom font avancer des principes importants liés à l'acquisition d'une L2. Dans le cadre de ce projet, nous avons choisi de souligner d'abord la distinction entre l'apprentissage et l'acquisition d'une deuxième langue, qui est une thèse avancée par Krashen (1982). Nous nous sommes également appuyées sur les propos de Tabors (2008), qui suggèrent quatre grandes étapes liées au processus d'acquisition d'une L2 chez les jeunes enfants de 4 et 5 ans.

Nous avons adopté les idées de Krashen et de Tabors pour former le cadre théorique de ce projet, car elles mettent l'accent sur les processus inhérents à l'apprenant ou à l'apprenante dans l'acquisition d'une langue seconde et non sur les facteurs externes (p. ex. contextes sociaux, stratégies d'enseignement, programmes d'études, etc.) qui peuvent influencer cette acquisition. Par ailleurs, ces idées nous fournissent des pistes intéressantes quant au développement bilingue des jeunes enfants dans le contexte des écoles francophones en milieu minoritaire.

\section{La distinction entre l'apprentissage et l'acquisition (Krashen, 1982)}

Selon Krashen, il existe une différence nette entre l'acquisition et l'apprentissage d'une deuxième langue. Pour lui, l'acquisition d'une L2 ressemble beaucoup à la façon dont les gens acquièrent une langue première, c'est-à-dire qu'il s'agit d'un processus amorcé dans le subconscient et propulsé par l'utilisation de la langue pour communiquer (Krashen, 1982). Il en résulte une "compétence acquise» et non apprise (Krashen, 1982, p. 10). De l'autre côté, l'apprentissage d'une L2 est un processus conscient par lequel les gens se familiarisent avec les aspects grammaticaux, syntaxiques et lexicaux de la langue. Si les deux processus jouent un rôle majeur dans le développement de la L2, le premier est considéré comme central et le deuxième comme périphérique. 
Après les constats de Krashen qui priorisent le processus d'acquisition, Tabors (2008) nous propose quatre stades d'acquisition d'une deuxième langue. Bien que ces stades soient présentés ici de façon séquentielle, il est important de garder à l'esprit le fait que les enfants ne sautent pas d'une étape à une autre, mais intègrent graduellement des éléments de l'étape suivante tout en maintenant des éléments de l'étape précédente pendant un certain temps.

\section{Les stades d'acquisition d'une deuxième langue selon Tabors (2008)}

Tabors (2008) estime qu'il existe quatre étapes dans le processus d'apprentissage d'une L2 chez les enfants: 1) utilisation persistante de la première langue; 2) période non verbale; 3) utilisation publique de la L2; 4) utilisation productive de la L2.

\section{L'utilisation persistante de la première langue}

Au début de la période de contact entre deux langues, certains enfants continuent d'utiliser leur première langue même si elle est incomprise. La durée de cette période varie de quelques jours à plusieurs mois. Tôt ou tard, les enfants dont personne dans la classe ne partage la langue finissent par réaliser que les gens autour d'eux ne les comprennent pas et qu'ils ne feront pas l'effort d'apprendre leur langue. Habituellement, cet état des choses pousse l'enfant à vouloir apprendre la nouvelle langue.

Nous avançons ici l'idée que dans le contexte minoritaire francophone, où une très grande majorité des personnes comprennent la première langue des apprenants (c.-à-d. l'anglais), il soit possible que les enfants persistent beaucoup plus longtemps dans cette phase.

\section{La période non verbale}

La période non verbale est caractérisée par l'absence de communication verbale. Malgré le fait que cette phase implique une période prolongée de silence de la part des apprenants de la langue, elle n'exclut pas toute forme de communication. Les enfants vont, par exemple, 1) montrer des objets pour attirer l'attention, 2) faire des gestes, 3) émettre des sons et 4) amorcer des jeux qui ne requièrent pas de mots. De plus, les enfants commencent à colliger l'information au sujet de la L2 principalement par deux stratégies: l'écoute attentive et la répétition.

Tabors (2008) établit une distinction entre la manifestation de cette phase au sein d'une classe monolingue, où personne ne parle la langue première de l'apprenant, et une classe bilingue, où d'autres enfants parlent la langue de l'apprenant. Dans le 
premier cas, l'enfant arrêtera de parler complètement. Dans l'autre, il arrêtera de parler à ceux qui ne parlent pas sa langue, mais continuera avec ceux qui la connaissent.

\section{L'utilisation publique de la L2}

Lorsque les enfants commencent leur utilisation publique de la langue, on remarque deux caractéristiques particulières: l'usage d'un langage télégraphique et d'un langage fait de préformulations (formulaic speech). Le langage télégraphique correspond à l'utilisation de quelques mots en remplacement d'une phrase complète, tandis le langage fait de préformulations consiste en l'utilisation de bouts de phrases préconstruits dans des situations où d'autres personnes les ont utilisés. Ces deux comportements langagiers sont typiques non seulement lors de l'apprentissage d'une L2, mais aussi chez les très jeunes enfants qui apprennent leur langue maternelle.

\section{L'utilisation productive de la $\mathrm{L} 2$}

Après avoir appris un nombre suffisant de mots de vocabulaire et de phrases utiles, les enfants peuvent commencer l'usage productif du langage. Ils commencent à construire leurs propres phrases au lieu de simplement répéter celles des autres. Pendant ce processus, les enfants doivent analyser la langue utilisée autour d'eux et deviner de quelle façon cette langue est construite. Typiquement, ils utilisent tout ce qu'ils connaissent au sujet de la nouvelle langue et font beaucoup d'erreurs jusqu'à ce qu'ils saisissent les aspects plus compliqués de la langue.

\section{MÉTHODOLOGIE DE RECHERCHE}

Puisque notre recherche avait pour objectif de mieux comprendre les comportements langagiers des élèves et d'en savoir davantage sur l'acquisition du français en contexte minoritaire dans le cadre de la francisation, nous avons opté pour une méthodologie qualitative. En gros, nous voulions observer les comportements langagiers des élèves pour voir quand et comment le français et l'anglais étaient employés par les élèves, ainsi que pour noter si ces comportements changeaient avec le temps.

Les questions de recherche étaient les suivantes: 1) Comment les comportements langagiers des élèves anglodominants et des élèves francodominants se manifestentils dans une classe maternelle de langue française? 2) Constate-t-on un changement dans ce comportement des élèves entre deux temps d'observation? 


\section{Contexte de l'étude}

En raison de sa situation géographique et de la forte influence de l'anglais sur son territoire, nous avons choisi le District scolaire francophone Sud (DSFS) au NouveauBrunswick pour faire cette collecte de données. Le DSFS couvre la partie centre-sud de la province et dessert la population francophone de cette région.

Nous avons ciblé trois classes de maternelle dans trois écoles différentes, situées respectivement dans des milieux de vitalité linguistique faible, moyenne et forte. Aux fins de cette description, nous nommerons les écoles participantes écoles A, B et C. À l'intérieur des trois classes maternelles de ces écoles, la proportion d'élèves anglodominants variait. La classe de l'école A comptait $19 \%$ d'élèves anglodominants, la classe de l'école B, $21 \%$ et la classe de l'école C, $43 \%$. Cet article présente l'analyse des données de l'école A seulement.

\section{Participantes et participants}

Dans le cadre de cette étude qualitative, l'échantillonnage s'est fait de façon délibérée (Karsenti et Savoie-Zajc, 2004; Patton, 2015), puisque nous voulions examiner les interactions de deux élèves anglodominants et de deux élèves francodominants de chacune des classes. La petite taille de cet échantillon se justifie, car la recherche qualitative repose sur une perspective idéographique, selon laquelle on cherche à comprendre un phénomène et non à l'expliquer (Robinson, 2014).

Avant son arrivée à l'école, la chercheure ${ }^{2}$ a envoyé des feuilles de consentement en français et en anglais aux enseignantes ainsi qu'aux parents de tous les élèves des classes participantes. Ensuite, elle a établi les critères de sélection suivants afin de guider ses choix d'élèves cibles: 1) Les élèves cibles étaient ceux et celles pour qui les parents avaient signé une feuille de consentement; 2) Les élèves cibles francodominants étaient ceux et celles qui parlaient français le plus souvent à la maison; 3) Les élèves cibles anglodominants étaient ceux et celles qui parlaient anglais le plus souvent à la maison.

Par la suite, la chercheure a recueilli de l'information des enseignantes participantes, qui avaient déjà signalé les élèves anglodominants de leurs classes. Cette information a accéléré le processus du choix des élèves cibles. Par conséquent, après une brève période d'observation lors de la première visite, la chercheure a pu désigner les quatre élèves cibles de chaque classe.

2. La collecte de données a été effectuée par la première auteure de cet article. 


\section{Collecte de données}

Deux séances de collecte de données de deux jours consécutifs ont été tenues dans chacune des trois écoles, la première à l'automne 2015 et la seconde au printemps 2016. La collecte de données était conçue de façon à recueillir le plus d'information possible au sujet des comportements langagiers des élèves. Afin d'observer ces comportements sans intervenir directement avec les enfants et de permettre à ceux-ci de s'exprimer librement et de façon naturelle, nous avons adapté la méthode employée par Ervin-Tripp (2000), Reyes (2004), Hickey (2007), Tabors (2008) et d'autres, qui ont utilisé des magnétophones individuels pour enregistrer les conversations des enfants. Pendant deux périodes de 20 minutes chacune, tous les élèves de la classe ont ainsi porté un petit magnétophone dans une pochette/ceinture et un microphone. Seules les interactions des quatre enfants ciblés ont été enregistrées. Nous avons choisi de donner des pochettes/ceintures à tous les élèves afin de protéger l'anonymat.

Deuxièmement, durant la journée entière, la chercheure a pris en note ses observations des comportements langagiers des élèves. Des entretiens informels et ponctuels ont été menés auprès du personnel enseignant dans le but de clarifier le contexte scolaire des élèves.

De plus, un questionnaire papier inspiré du travail de Landry, Allard et Deveau (2010) a été établi par les chercheures et envoyé à la maison à l'intention des parents. Ce questionnaire, qui comprenait deux sections, avait pour but de dégager de l'information pertinente au sujet de l'héritage culturel des enfants et de leurs comportements langagiers à l'extérieur de l'école. La section au sujet des comportements langagiers comprenait seize questions du style Likert. Voici un exemple de question posée: Quelle(s) langue(s) votre enfant utilise-t-il avec les personnes suivantes: père, mère, grands-parents paternels, grands-parents maternels, etc.? Dans cet exemple, les choix de réponses étaient les suivants: Presque toujours le français, le français un peu plus que l'anglais, le français et l'anglais également, l'anglais un peu plus souvent que le français, presque toujours l'anglais. L'analyse des sondages des parents n'est pas présentée dans cet article, car l'important ici est de cibler les comportements langagiers des élèves à l'école.

\section{Dépouillement des données}

Les enregistrements audio ont été transcrits et les notes d'observation ont été mises sous forme numérique. Une première lecture globale du corpus a permis de repérer les zones ou situations de la classe où les comportements langagiers des élèves se manifestaient. Des codes ont été créés pour décrire ces zones/situations (Feng, Foo, Kretschmer, Prendeville et Elgas, 2004), telles que "dans le cercle», "dans les centres», «dans des activités structurées d'apprentissage», «avec les adultes», «avec 
les élèves anglodominants» et "avec les élèves francodominants». Ces codes ont été établis pour les élèves anglodominants et les élèves francodominants. Nous avons ensuite fait une relecture du corpus afin de déterminer les comportements langagiers qui se manifestaient chez les élèves anglodominants et francodominants à l'intérieur de ces zones/situations. Pour terminer, de façon globale, nous avons comparé les données des deux temps d'observation afin de voir si un changement avait eu lieu entre les deux moments. Le logiciel NVivo 11 a servi au codage des données.

\section{RÉSULTATS}

Cette partie présente les résultats découlant de l'analyse de nos données en vue de répondre aux questions de recherche.

\section{Élèves anglodominants en novembre 2015}

À l'école A, quatre élèves sur vingt et un étaient reconnus comme élèves anglodominants. À la première période d'observation, un constat important fut que ces élèves parlaient très peu. D'après nos observations, et selon l'information fournie par la titulaire de la classe, les élèves anglodominants parlaient le plus souvent entre eux, et uniquement en anglais. Lorsqu'il arrivait à ces élèves de parler avec des élèves francodominants, ils le faisaient également en anglais. Cependant, ces échanges ont été plutôt rares. En ce qui a trait à la communication verbale avec les adultes dans la classe (enseignante titulaire, assistante en éducation, surveillante, etc.), celle-ci était amorcée par les adultes seulement, dans un effort de faire parler les élèves.

Les comportements langagiers des élèves anglodominants observés à l'intérieur des différentes zones de la classe montrent ces mêmes tendances. Par exemple, lors des moments dans le cercle, lorsque les élèves étaient tous assis par terre devant l'enseignante qui animait une discussion de groupe, la communication verbale de la part des élèves anglodominants était excessivement rare. En fait, la chercheure n'a pas pu observer un seul élève anglodominant parler de façon spontanée dans le cercle pendant les deux jours d'observation en novembre. Il en est de même pour les moments d'activités structurées d'apprentissage, où les élèves avaient à accomplir individuellement des tâches à leur pupitre. Là encore, les élèves anglodominants étaient très silencieux. La communication de ces élèves se faisait plutôt par le non-verbal (c.-àd. des expressions du visage, des mouvements de la tête, des épaules, etc.).

Dans les centres d'apprentissage, les comportements langagiers des élèves anglodominants variaient quelque peu de cette norme. Le temps des «centres" était un moment de jeu libre où les élèves pouvaient choisir leurs activités et les camarades avec qui ils ou elles voulaient jouer. 
Ces moments étaient très fugaces, car les élèves étaient portés à changer d'activités et de compagnons de jeu souvent et rapidement. C'est grâce à ces moments que nous avons pu observer l'interaction sociale entre les élèves anglodominants et les autres. La plupart du temps, ces élèves jouaient entre eux, et les interactions étaient uniquement en anglais. Il y a eu quand même quelques moments d'interaction entre les élèves anglodominants et les élèves francodominants. Lors de ces interactions, les élèves anglodominants ne parlaient qu'en anglais, même si l'élève francodominant parlait en français.

En considérant, à la lumière des propos de Tabors (2008), les comportements langagiers des élèves anglodominants en novembre, nous pensons qu'à ce moment de l'année il se peut que les élèves anglodominants chevauchent les deux premiers stades, soit l'utilisation persistante de la langue première et la période non verbale. Il est vrai que les élèves anglodominants ne faisaient aucune communication verbale devant le groupe-classe et qu'ils choisissaient de parler presque uniquement avec d'autres élèves anglodominants. De plus, des comportements non verbaux associés à la période non verbale, tels que la communication non verbale et des périodes prolongées d'observation, furent constatés. Par ailleurs, un des élèves anglodominants s'exerçait à insérer du vocabulaire français dans ses énoncés. L'enregistrement audio a capté l'échange suivant entre deux élèves anglodominants:

Enfant 1: «Fini» the card. Do another card. I'm doing this. Everything on mine will be green. Everything will be green to me.

Enfant 2: Why do you like green so much?

Enfant 1: «Vert» is my favourite colour.

Enfant 2: Blue is my favourite colour. And yellow.

Enfant 1: You mean «bleu».

Il importe de mentionner que les enfants qui en sont à cette étape d'acquisition de la langue ne comprennent pas tout ce qui est dit autour d'eux. Cependant, dans le contexte d'une salle de classe bien structurée, ces enfants peuvent suivre et imiter ce que les autres font, et ils peuvent donner l'impression de bien comprendre (Tabors, 2008).

Un exemple provenant des enregistrements audio sert à renforcer ce constat. Lors d'un moment de jeu dans les centres, lorsque l'enseignante travaillait avec un petit groupe d'élèves, nous avons capté la voix de l'enseignante, puis la réaction d'un élève anglodominant: 
Enseignante: Les amis en arrière, c'est très, très fort. Surtout les filles dans les déguisements et [nom d'un enfant]. Madame [son nom] essaie de travailler avec des amis et elle ne les entend pas parler.

Enfant 1: [Qui parle à l'enfant à côté de lui] Not time to "ranger», right? We don't need to clean up, right?

Ici nous suggérons que l'enfant anglodominant ne comprend pas ce que l'enseignante dit et qu'il essaie de confirmer auprès de son ami s'il a bien compris la consigne.

De ces constats, nous déduisons que les quatre élèves anglodominants de cette classe étaient tous plus ou moins parvenus au stade de la période non verbale. De plus, ils représentaient un groupe plutôt homogène, car leurs comportements langagiers se ressemblaient beaucoup.

\section{Élèves francodominants en novembre 2015}

En novembre, les élèves francodominants parlaient librement en français entre eux et avec les adultes, et ce, dans toutes les zones de la classe. Dans le cercle, les élèves participaient aux discussions en levant la main, ils adressaient des commentaires à d'autres et interagissaient pleinement avec l'enseignante. Lors des activités structurées, ils discutaient avec d'autres en posant des questions et en faisant des commentaires. Dans toutes ces situations, il était évident que ces élèves ne se trouvaient pas en situation d'acquisition de la langue et étaient donc libres d'explorer leur environnement scolaire en français avec enthousiasme et intérêt.

Lorsque les élèves francodominants parlaient avec les élèves anglodominants, nous avons observé plusieurs différents comportements langagiers. En voici quelques exemples. À un moment où deux élèves anglodominants jouaient avec un seul enfant francodominant, le jeu se passait en anglais. Dans une situation où deux élèves francodominants jouaient avec un élève anglodominant, le jeu se passait en français. Dans la situation où un élève francodominant et un élève anglodominant jouaient ensemble, l'anglais ou un mélange d'anglais et de français ont été employés. Cependant, même si toutes ces situations ont été observées durant les deux premiers jours d'observation, la plupart du temps les élèves anglodominants jouaient entre eux, tandis que les élèves francodominants faisaient de même de leur côté.

D'après ces constats, nous remarquons que les élèves francodominants étaient portés à choisir l'anglais dans leurs interactions avec les élèves anglodominants à ce moment de l'année. Cependant, il s'agissait généralement d'interactions de courte durée après lesquelles la conversation reprenait en français. 


\section{Élèves anglodominants en avril 2016}

Nous sommes retournées à cette classe en avril 2016 afin de constater s'il y avait eu des changements par rapport aux comportements langagiers des enfants. En ce qui a trait aux élèves anglodominants en tant que groupe, nous avons remarqué une augmentation des incidences d'interaction sociale et un progrès important dans l'utilisation du français chez ces élèves.

À ce moment de l'année, les élèves anglodominants montraient des différences observables dans leurs comportements langagiers et n'agissaient donc plus en tant que groupe homogène. Certes, les facteurs individuels qui influencent l'acquisition d'une langue seconde (L2), tels que la motivation, la personnalité, le niveau d'exposition à la langue et l'âge, sont bien documentés dans la recherche (Tabors, 2008). Pour illustrer ces divergences dans les comportements langagiers des élèves, nous prendrons l'exemple des deux élèves anglodominants que nous avons observés dans cette classe. En examinant de plus près les comportements langagiers de ces élèves en avril, nous constatons des différences importantes.

D'abord, les comportements langagiers du premier élève avaient peu changé depuis le mois de novembre. Lorsqu'il parlait, il employait encore l'anglais la plupart du temps. Dans le cercle ou durant les activités structurées, il demeurait silencieux et passait de longues périodes à observer et à écouter les conversations des autres. Avec les adultes, il amorçait très peu la communication. Ces derniers continuaient à l'encourager pour qu'il communique verbalement. Dans ces situations, il misait fortement sur le non-verbal, tout comme en novembre. D'après ces observations, cet élève se trouvait selon nous encore plus ou moins dans la période non verbale.

En revanche, nous avons remarqué plusieurs changements dans les comportements langagiers du deuxième élève. D'abord, avec les différents groupes d'interlocuteurs, cet élève amorçait la conversation beaucoup plus souvent qu'en novembre. Avec les adultes et les élèves francodominants, il parlait sans incitation, en faisant l'effort de parler en français et en misant beaucoup sur les préformulations et le langage télégraphique.

Comme nous l'avons vu, le langage fait de préformulations consiste de bouts de phrase préconstruits (Tabors, 2008). Quelques exemples de préformulations utilisées par ce jeune apprenant de la langue étaient «Regarde, Madame!», "J'ai fini!» et «Nomme-moi!». Pour ce qui est de l'utilisation du langage télégraphique, lors des activités structurées, il a dit: «Moi faire tout ça» (en montrant la page qu'il venait de compléter) et "Les cinq, c'est bien» (en voulant dire que les chiffres qu'il avait écrits étaient bien formés). Durant les centres, il a employé le langage télégraphique avec d'autres élèves. À plusieurs reprises, il s'est approché d'autres élèves dans le but de participer à leur jeu en disant "Moi jouer?». Plus tard, en jouant aux camions avec trois élèves francodominants, il a dit: «Moi c'est méchant. Moi c'est méchant, ok?» en 
montrant son camion aux autres. Ensuite, il a pointé du doigt le camion d'un autre élève en disant "C'est gentil!». Les élèves ont ensuite fait une bataille entre les camions méchants et gentils, en français, pendant cinq minutes.

Une dernière stratégie d'acquisition de la langue observée chez cet élève était celle de la répétition. Selon Tabors (2008), la répétition consiste en des verbalisations de la part de l'enfant qui sont une manière de se familiariser avec les sons et aux structures de la langue. La plupart du temps, ces verbalisations sont faites à voix basse et ne sont pas faciles à entendre. Puisque nous avons employé des magnétophones, nous avons pu capter certaines répétitions que faisait cet élève. Dans une situation où il jouait seul, nous l'avons enregistré disant: "Couper. Couuuuuper. Couper, couper couper. Ciseaux, ciiiiiseaux» (en chantonnant).

Ensuite, à deux reprises, nous l'avons observé en train de chanter tout bas des chansons familières (La chanson de l'alphabet et Bonne fête) qui lui permettaient d'améliorer son français.

Dans les différentes zones de la classe et avec une variété d'interlocuteurs, il est évident que cet élève était prêt à prendre des risques en ce qui a trait à la communication verbale. Dans le cercle, malgré le fait qu'il demeurait très silencieux, il a tout de même levé la main pour parler à deux reprises durant ce deuxième temps d'observation. Il est le seul des élèves anglodominants à avoir levé la main durant les quatre jours d'observation dans cette classe. Par ailleurs, lors d'un moment de jeux libres, nous l'avons observé qui participait à une conversation en français au sujet du hockey avec deux élèves francodominants. Enfin, au cours d'une activité structurée où les élèves s'entraînaient à écrire la lettre " $G$ » dans leur cahier, cet élève a fait une blague en faisant semblant de faire un "X» sur sa page. En exagérant ses mouvements et en faisant une grimace, il a dit "Un gros " $x$ "! . Les autres élèves à sa table ont réagi en riant.

Il est vrai que la stratégie de la répétition, ainsi que les moments prolongés d'observation, est conforme au stade 2 de Tabors (2008) et que l'utilisation du langage télégraphique et du langage préformulé est associée au stade 3 . À la lumière de ces observations, nous supposons alors que cet élève avait progressé dans les stades d'acquisition de la langue depuis le mois de novembre et qu'il chevauchait les stades 2 et 3 .

\section{Élèves francodominants en avril 2016}

En avril, les élèves francodominants continuaient à utiliser le français comme ils le faisaient en novembre avec les adultes, dans le cercle et lors des activités structurées. Un changement observable s'est produit en ce qui a trait aux interactions des élèves francodominants avec les élèves anglodominants. Rappelons-nous qu'en novembre 
les élèves francodominants choisissaient la plupart du temps l'anglais pour interagir avec les élèves anglodominants. En avril, la langue d'interaction entre les élèves était le français ou bien un mélange d'anglais et de français. Qui plus est, tandis qu'en novembre les interactions entre les élèves anglodominants et les élèves francodominants étaient plutôt restreintes, en avril ces élèves jouaient et discutaient beaucoup plus ensemble.

\section{DISCUSSION}

L’objectif primaire de cette étude était d'observer les comportements langagiers des élèves dans une maternelle francophone où la francisation était présente, dans le but de mieux comprendre ces comportements et de voir s'ils changeaient entre deux temps d'observation. Dans le contexte de l'école A de cette étude, où le nombre d'élèves anglodominants était restreint (4 élèves sur 21), nos résultats suggèrent que ces élèves semblent suivre les stades d'acquisition de Tabors (2008), se situant, en novembre, dans une période plutôt silencieuse. À ce deuxième stade d'acquisition, les élèves anglodominants de cette classe ne parlaient que très peu. Et lorsqu'ils se trouvaient à communiquer verbalement, c'était uniquement en anglais et surtout avec les élèves anglodominants. En revanche, les élèves anglodominants suivaient très bien la routine de la classe et misaient fortement sur le non-verbal dans leurs interactions avec d'autres.

Plusieurs changements ont été remarqués chez ces élèves entre les deux temps d'observation. D'abord, les élèves anglodominants cheminaient dans leur acquisition langagière à des vitesses variées. De plus, alors que ce groupe était plutôt silencieux en novembre, il y avait davantage de moments de prise de parole et de tentatives de production langagière en avril. Chez un élève en particulier, nous avons remarqué des comportements qui correspondent au stade 3 décrit par Tabors (2008), dont l'usage du langage télégraphique et des préformulations.

À notre avis, deux autres constats importants de cette étude méritent des investigations futures. D'abord, en regardant les comportements langagiers à l'intérieur des différentes zones de la salle de classe, il nous apparaît que ces zones semblent exercer une certaine influence sur la quantité de prise de parole des élèves anglodominants. Par exemple, tandis que ces élèves étaient très silencieux dans le cercle pendant les deux temps d'observation, ils faisaient beaucoup plus de tentatives pour parler français dans les jeux libres et au cours des activités d'apprentissage structurées, surtout en avril. Il apparaitt important d'examiner si ces tendances se reproduisent chez d'autres élèves anglodominants dans d'autres salles de classe et de mieux comprendre les implications pédagogiques de celles-ci.

Dans le contexte de cette salle de classe, qui se situait dans une école au sein d'une communauté francophone de vitalité plutôt forte, nous avons également remarqué 
que l'attirance pour la langue française était forte elle aussi (Landry, Allard et Deveau, 2010). En d'autres mots, les élèves francodominants parlaient presque uniquement en français lors des deux moments d'observation et les comportements langagiers des élèves anglodominants intégraient progressivement de plus en plus d'énoncés en français. Les analyses subséquentes des données des deux autres écoles, c'est-àdire celle qui se situe dans un milieu de vitalité moyenne et celle qui se trouve dans un milieu de vitalité faible, nous permettront de voir si ces dispositions se maintiennent dans des contextes sociaux où le contact entre l'anglais et le français est plus prononcé.

\section{CONCLUSION}

Ainsi que nous l'avons précisé en introduction, environ $50 \%$ des élèves admissibles à l'école de langue française en contexte minoritaire n'ont pas le français comme langue maternelle. Il est donc important de s'intéresser à ces élèves et d'observer comment leurs habiletés langagières se développement à l'école. Les résultats de cette étude éclairent donc davantage le phénomène de francisation en tant que processus d'acquisition d'une langue seconde en contexte linguistique minoritaire. Puisque les élèves qui ont participé à cette recherche semblent suivre les stades d'acquisition d'une langue seconde définis par Tabors (2008), il serait intéressant de réfléchir à des actions pédagogiques spécifiques à proposer pour soutenir les élèves anglodominants à leurs divers stades d'acquisition langagière. Pour les élèves francodominants, qui subissent également l'influence anglodominante de leur milieu, il s'agirait de continuer d'assurer le meilleur cheminement langagier possible.

Pour terminer, considérant que des liens plausibles peuvent être établis entre les propos de Tabors (2008) et le contexte de l'école de langue française en milieu minoritaire, il nous paraît raisonnable de conclure que d'autres concepts provenant du domaine du bilinguisme pourraient appuyer les efforts des intervenants et intervenantes scolaires dans les écoles francophones. À notre avis, dans un système scolaire chargé d'assurer le développement du français, tant chez des élèves anglodominants que chez des francodominants, une meilleure compréhension du développement bilingue des élèves pourrait mieux outiller le personnel enseignant qui doit répondre aux besoins variés de tous. 


\section{Références bibliographiques}

ARSENAULT-CAMERON, C. Île-du-Prince-Édouard [2000] 1 RCS 3.

CHANDLER, V. (2017, mars). Les dommages causés par les réparations. Astheure. Repéré à https://astheure.com/2017/03/22/les-dommages-causes-par-les-reparationsvincent-chandler/

COMMISSAIRE AUX LANGUES OFFICIELLES. (2000). La mission de l'instruction dans la langue de la minorité. Présentation devant les États généraux sur la petite enfance. Repéré à http://www.languesofficielles.gc.ca/html/speeches_discours_ 29012000_f.php

CONSEIL DES MINISTRES DE L'ÉDUCATION (CANADA). (2002). La francisation: pour un état des lieux. Toronto: Conseil des ministres de l'Éducation (Canada). Repéré à http://francisation.cmec.ca/documents/00b_Pourunetatdeslieux.pdf

CORMIER, M. (2015). Accueillir le majoritaire dans l'institution de la minorité? Minorités linguistiques et société / Linguistic Minorities and Society, 5, 100-121. doi: 10.7202/1029109ar

CORMIER, M., BOURQUE, J. et JOLICOEUR, M. (2014). (Re)-introduction to French: Four education models to revitalise an endangered group in Eastern Canada. International Journal of Bilingual Education and Bilingualism, 17(2), 160-177. doi: 10.1080/13670050.2013.866626

CORMIER, M. et LOWE, A. (2010). La francisation dans les écoles du NouveauBrunswick: défis et moyens. Dans L. Arrighi, M. Leblanc et L. Péronnet (dir.), La francophonie en Acadie: dynamiques sociales et langagières. Textes en hommage à Louise Péronnet (p. 223-245). Sudbury, Ont. : Prise de paroles.

ERVIN-TRIPP, S. M. (2000). Studying conversation: How to get natural peer interaction. Dans L. Menn et N. B. Ratner (dir.), Methods of studying language production (p. 271-288). Hillsdale, NJ: Lawrence Erlbaum Associates.

FARMER, D. (2008). L'immigration francophone en contexte minoritaire: entre la démographie et l'identité. Dans J.-Y. Thériault, A. Gilbert et L. Cardinal (dir.), L'espace francophone en milieu minoritaire au Canada: nouveaux enjeux, nouvelles mobilisations (p. 121-159). Montréal: Fides.

FENG, S., FOO, S. F., KRETSCHMER, R., PRENDEVILLE, J. et ELGAS, P. M. (2004). Language and peer culture: Mandarin-speaking preschoolers in an English dominant setting. Language and Education, 18(1), 17-34. doi: 10.1080/ 09500780408666865 
GALLANT, N. et DENIS, W. (2008). Relever le défi de la diversité: une comparaison des idéologies en éducation en contexte minoritaire et majoritaire au NouveauBrunswick et en Saskatchewan. Éducation et francophonie, XXXVI(1), 142-160.

GÉRIN-LAJOIE, D. (2006). La contribution de l'école au processus de construction identitaire des élèves dans une société pluraliste. Éducation et francophonie, 34(1), 1-7.

GILBERT, A., LETOUZÉ, S., THÉRIAULT, J.-Y. et LANDRY, R. (2004). Le personnel enseignant face aux défis de l'enseignement en milieu minoritaire francophone. Rapport final de recherche. Ottawa: Fédération canadienne des enseignantes et des enseignants.

HELLER, M. (2011). Paths to post-nationalism. A critical ethnography of language and identity. Oxford: Oxford University Press.

HICKEY, T. M. (2007). Children's language networks in minority language immersion: What goes in may not come out. Language and Education, 21(1), 46-65, doi: $10.2167 / \mathrm{le} 617.0$

KARSENTI, T. et SAVOIE-ZAJC, L. (2004). La recherche en éducation: étapes et approches. ( $3^{\mathrm{e}}$ éd.). Sherbrooke: Éditions du CRP.

KRASHEN, S. D. (1982). Principles and practice in second language acquisition. Oxford, R.-U.: Pergamon Press.

LANDRY, R. (2003). Libérer le potentiel caché de l'exogamie. Profil démolinguistique des enfants des ayants droit francophones selon la structure familiale. Moncton, N.-B.: Institut canadien de recherche sur les minorités linguistiques et Ottawa: Commission nationale des parents francophones.

LANDRY, R. (2006). Ayants droit et école de langue française: le cas de l'exogamie. The Supreme Court Law Review, 23, 149-171.

LANDRY, R. (2010). Petite enfance et autonomie culturelle: là où le nombre le justifie... $V$, Moncton, N.-B.: Institut canadien de recherche sur les minorités linguistiques.

LANDRY, R. et ALLARD, R. (1997). L'exogamie et le maintien de deux langues et de deux cultures: le rôle de la francité familioscolaire. Revue de sciences de l'éducation, 23(3), 561-592.

LANDRY, R., ALLARD, R. et DEVEAU, K. (2010). École et autonomie culturelle. Enquête pancanadienne en milieu scolaire francophone minoritaire. Ottawa: Institut canadien de recherche sur les minorités linguistiques. 
MARTEL, A. (2001). Droits, écoles et communautés en milieu minoritaire: 1986-2002. Analyse pour un aménagement du français par l'éducation. Ottawa: Commissariat aux langues officielles.

PATTON, M. Q. (2015). Qualitative research and evaluation methods. (4 éd.). Thousand Oaks, CA: Sage.

REYES, I. (2004). Functions of code switching in schoolchildren's conversations. Bilingual Research Journal, 28(1), 77-98.

ROBINSON, O. C. (2014). Sampling in interview-based qualitative research: A theoretical and practical guide. Qualitative Research in Psychology, 11(1), 25-41. doi: $10.1080 / 14780887.2013 .801543$

ROCQUE, J. (2006a). L'éducation en français langue première:étude sur le phénomène de l'exogamie et la gestion scolaire en milieu minoritaire - une étude de cas du Conseil scolaire Centre-est de l'Alberta (Thèse de doctorat non publiée). Université Laval, Québec.

ROCQUE, J. (2006b). Vers l'élaboration d'une politique de l'exogamie dans le cadre de la gestion scolaire francophone en milieu minoritaire. Revue de la Common Law en français, 8, 121-153.

ROCQUE, J. (2010-2011). Phénomène de l'exogamie dans le cadre de la gestion scolaire francophone en milieu minoritaire: constats et interventions. Revue du Nouvel-Ontario, 35-36, 189-211.

SAVILLE-TROIKE, M. (2006). Introducing second language acquisition. Cambridge, R.-U.: Cambridge University Press.

TABORS, P. O. (2008). One child, two languages. A guide for early childhood educators of children learning English as a second language. Baltimore, MD: Paul H. Brookes. 